\title{
On the mechanism for breaks in the cosmic ray spectrum
}

\author{
M.A. Malkov ${ }^{1}$, P.H. Diamond ${ }^{1,3}$, R.Z. Sagdeev ${ }^{2}$ \\ ${ }^{1}$ CASS and Department of Physics, University of California, \\ San Diego, La Jolla, CA 92093 \\ ${ }^{2}$ University of Maryland, College Park, MD 20742-3280 \\ ${ }^{3}$ National Fusion Research Institute, Daejeon, Korea
}

\begin{abstract}
The proof of cosmic ray (CR) origin in supernova remnants (SNR) must hinge on full consistency of the CR acceleration theory with the observations; direct proof is impossible because of the orbit stochasticity of CR particles. Recent observations of a number of galactic SNR strongly support the SNR-CR connection in general and the Fermi mechanism of CR acceleration, in particular. However, many SNR expand into weakly ionized dense gases, and so a significant revision of the mechanism is required to fit the data. We argue that strong ion-neutral collisions in the remnant surrounding lead to the steepening of the energy spectrum of accelerated particles by exactly one power. The spectral break is caused by a partial evanescence of Alfven waves that confine particles to the accelerator. The gamma-ray spectrum generated in collisions of the accelerated protons with the ambient gas is also calculated. Using the recent Fermi spacecraft observation of the SNR W44 as an example, we demonstrate that the parent proton spectrum is a classical test particle power law $\propto E^{-2}$, steepening to $E^{-3}$ at $E_{b r} \approx 7 \mathrm{GeV}$.
\end{abstract}

\section{INTRODUCTION}

The discovery of cosmic rays (CR) dates back to the historic Victor Hess balloon ascent in 1912 [1]. CR origin is thus a century old problem. Only the latest direct observations of galactic supernova remnants (SNRs) [2-9] narrowed the search to precisely these objects as the most probable sources of the CRs. One serious problem on the observational side was the lack of the SNR gamma-ray data below the energy range of the imaging atmospheric Cerenkov telescopes, or IACT. The Fermi-LAT (large area telescope) and Agile observatories are rapidly bridging this gap (roughly in the 0.1-30 GeV band e.g., [4, 9]), virtually overlapping with the IACT energy band. There have been recent breakthrough observations of such SNR as W44, IC443, W28, RX J1713 and Cas A [4, 6-8, 10]. Overall, observations favor the diffusive shock acceleration (or DSA [11-13], a modern version of the mechanism originally suggested by Fermi in 1949 [14]) as a means for the production of galactic CRs. However, there are questions, and even some challenges, that the recent observations pose to the theory.

Of those, the most relevant to the proof of the SNR-CR connection is the form of the spectrum that the theory predicts for the particular SNR conditions. Full understanding of the spectra will allow one to disentangle the proton (i.e., the primary CR component) emission from a contaminating (1-2\% level) but radiatively more efficient, and accessible to the direct observations, electron CR component. The most recent challenge to the DSA was posed by the measurements of the rigidity (momentum to charge) spectra of different species (most notably proton and helium). They turned out to be different, contrary to the DSA predictions for the ultra-relativistic rigidity range.

Note that the latter problem arose from the indirect observations of the background CRs [15-18], as opposed to the above mentioned direct observations of the putative accelerators (SNR). Generally, it is impossible to trace CR back to their accelerators because of the orbit scrambling. The proof of their origin in SNRs can only be achieved by proving the acceleration theory consistent with all accessible observations. It should be noted that 'direct' observations also provide only the secondary photon emission generated by accelerated particles, either electrons (through synchrotron, Bremsstrahlung and inverse Compton radiation), or protons (through their collisions with the ambient gas material). Therefore, such observations cannot be interpreted as an evidence of proton acceleration in SNR without a detailed understanding of the emission mechanism. Note that electron acceleration in SNRs to at least $\sim 100 \mathrm{TeV}$ is held proven "beyond a reasonable doubt" after the observations of the SNR 1006 by ASCA and other X-ray instruments [19, 20].

This paper deals with the modification of the DSA proton spectra in a partially ionized SNR environment and its signatures in gamma-emission from such remnants. The recent discovery of the proton/helium anomaly in the background CR spectra is discussed elsewhere ([21], see also [22-24] for other suggestions to explain this anomaly). Here we pursue an alternative, complementary approach to more common multi-band treatments, e.g., [25], where the fits are primarily focused on the overall agreement across the entire spectrum (from radio to gamma). By contrast, we concentrate on the gamma-ray band and fit an important signature of the spectrum which is the spectral break. We believe it conveys an important information about the physics of acceleration missed in the 'standard' DSA theory. The quality of our fit, with virtually no adjustable parameters, should testify for the underlying physical scenario behind the emission. The broad-band fits do not typically meet high-quality criteria, as they seek to fit several portions of the data simultaneously by adjusting, in some cases, a few free parameters. Nevertheless, they provide an excellent consistency check for each particular model.

The recent Fermi-LAT observations of the SNRs W44 and IC443 [4, 7] indicate that the spectrum of the gamma ray producing protons is substantially steeper in its high energy part than the DSA predicts. A similar discrepancy has been found in the 
high energy gamma ray spectra measured by e.g., the CANGAROO [2], HESS [3] and MAGIC [26] atmospheric Cerenkov telescopes. The lack of understanding of the primary particle spectra triggered debates about the nature of the observed gammaray emission (hadronic vs leptonic), e.g., [27]. We argued [28] that when a SNR interacts with a dense molecular cloud complex, the conditions for particle confinement to the shock are different from those adopted in conventional DSA modeling. Since the propagation of resonant Alfven waves is inhibited by ion-neutral collisions, particles are not confined and so escape the emission volume. These phenomena should result in a spectral break in the parent proton and thus, in the gamma-ray spectrum. The spectral index at the break should change by exactly one power $\Delta q=1$ due to an effective reduction of particle momentum space dimension by one, since particles are confined in coordinate space only when they are within a slab in momentum space oriented perpendicular to the local mean magnetic field. Note that the earlier HESS observations of the SNR RXJ 1713 were also consistent with such a break [3]. The most convincing evidence for the breaks of index one, however, provide the recent Fermi-LAT and Agile observations of W44 [4, 9, 29] (re-analyzed in [30]), the MAGIC observations of the SNR W51C [26, 31] as well as the FERMI observations [32] of giant molecular clouds (GMC), where the Alfven wave evanescence should also result in a $\Delta q \simeq 1$ steepening of the $E^{-q} \mathrm{CR}$ primary spectrum. These observations are encouraging in that they unambiguously confirm the breaks. However, they rule out traditional DSA models based on a single power law with an exponential cutoff.

\section{MECHANISM FOR THE SPECTRAL BREAK}

The physics of the spectral break considered here is very simple. When a SNR shock approaches a molecular cloud (MC) or a pre-supernova swept-up shell, confinement of accelerated particles deteriorates. Due to the particle interaction with magnetic turbulence, confinement generally requires scales similar to the particle gyroradius [11, 12]. However, strong ion-neutral collisions substantially enhance the role of particle pitch angle in wave-particle interaction. While the waves are in a strongly ionized (closer to the shock) medium they propagate freely in a broad frequency range at the Alfven speed $V_{A}=B / \sqrt{4 \pi \rho_{i}}$ with the frequencies $\omega=k V_{A}$. Here $k$ is the wave number (assumed parallel to the local field $\mathbf{B}$ ) and $\rho_{i}$ is the ion mass density. As long as the Alfven wave frequency is higher than the ion-neutral collision frequency $v_{i n}$, the waves are weakly damped. When, on the other hand, the ion-neutral collision frequency is higher (deeper into the cloud), neutrals are entrained by the oscillating plasma and the Alfven waves are also able to propagate, albeit with a factor $\sqrt{\rho_{i} / \rho_{0}}<1$ lower speed, where $\rho_{0}$ is the neutral density. The propagation speed reduction occurs because every ion is now "loaded" with $\rho_{0} / \rho_{i}$ neutrals. In between these two regimes Alfven waves are heavily damped and even disappear altogether for sufficiently small $\rho_{i} / \rho_{0} \ll 0.1$. The evanescence wave number range is then bounded by $k_{1}=v_{i n} / 2 V_{A}$ and $k_{2}=2 \sqrt{\rho_{i} / \rho_{0}} v_{i n} / V_{A}$. These phenomena have been studied in detail in [33, 34], and specifically in the context of the DSA in [35-37]. Now we turn to their impact on the particle confinement and emissivity.

In the frame work of a quasilinear wave-particle interaction the wave number $k$ is approximately related to the parallel (to the magnetic field) component of the particle momentum $p_{\|}$by the cyclotron resonance condition $k p_{\|} / m= \pm \omega_{c}$, where the (non-relativistic) gyro-frequency $\omega_{c}=e B / m c$. Note that the appearance of $p_{\|}=p \mu$, where $\mu$ is the cosine of the pitch angle (see Fig 11), instead of the often used "sharpened" [38] resonance condition $\mathrm{kp} / \mathrm{m}= \pm \omega_{c}$ is absolutely critical for the break mechanism.

The frequency range where the waves cannot propagate may be conveniently translated into the parallel momentum range

$$
p_{1}<\left|p_{\|}\right|<p_{2},
$$

with

$$
p_{1}=2 V_{A} m \omega_{c} / v_{i n}, p_{2}=\frac{p_{1}}{4} \sqrt{\rho_{0} / \rho_{i}}>p_{1} .
$$

That a spectral break must form at the photon energy corresponding to the particle momentum $p=p_{1}=p_{b r}$, can be readily understood from Fig 1. The 'dead zones' $p_{1}<\left|p_{\|}\right|<p_{2}$ imply that particles with $\left|p_{\|}\right|>p_{1}$ do not turn around (while moving along the magnetic field) and escape from the region of CR-dense gas collisions at a $p_{\|} / p$ fraction of the speed of light. More specifically, particles with $p_{1}<\left|p_{\|}\right|<p_{2}$ escape because they are not scattered, whereas particles with $\left|p_{\|}\right|>p_{2}$, while being scattered, maintain the sign of $p_{\|}$, as they cannot jump over the gap $p_{1}<\left|p_{\|}\right|<p_{2}$ and so escape as well. An exception to this are particles with sufficiently large $p_{\perp}$ that can be mirrored across the gap or overcome it via the resonance broadening. We return to this possibility later.

The break can also be explained in terms of the confinement times of different groups of particles introduced above. Particles with $\left|p_{\|}\right|>p_{1}$ spend only short time $\tau_{\mathrm{esc}} \sim L_{\mathrm{c}} / c$ (where $L_{\mathrm{c}}$ is the size of the clump) inside the gas clumps. They propagate ballistically and their scattering time is assumed to be infinite, as there are no waves they can interact with resonantly $\left(p_{1}<\right.$ $\left.\left|p_{\|}\right|<p_{2}\right)$ or they cannot change their propagation direction $\left(\left|p_{\|}\right|>p_{2}\right)$. Particles with $\left|p_{\|}\right|<p_{1}$ are, on the contrary, scattered 


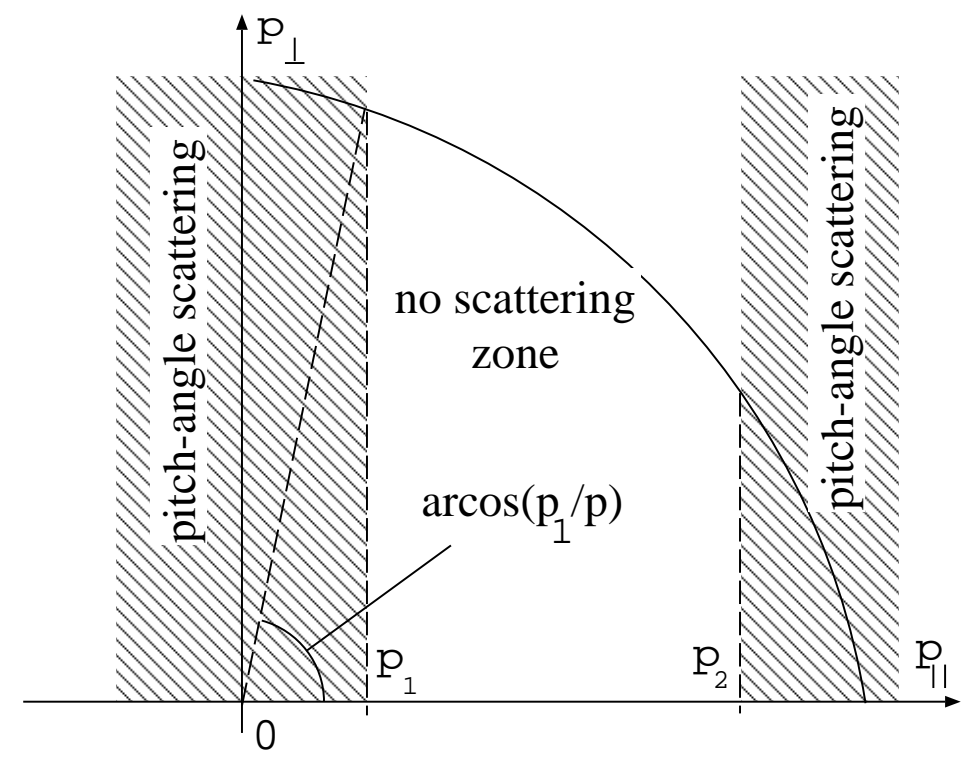

Figure 1: Momentum space of accelerated protons. Particle scattering zones on the $\left(p_{\|}, p_{\perp}\right)$ - plane of momentum space. Protons in the stripes $p_{1}<\left|p_{\|}\right|<p_{2}$ are not scattered by waves (see text). Therefore, particles from the domains $\left|p_{\|}\right|>p_{2}$ maintain their propagation direction and promptly escape from the dense gas region.

intensively in pitch angle, they frequently change their direction, and so sit in the clump for $\tau_{\text {conf }} \sim L_{\mathrm{c}}^{2} / \kappa \sim L_{\mathrm{c}}^{2} / c^{2} \tau_{\mathrm{sc}}$. Here $\tau_{\mathrm{sc}}$ is their pitch-angle scattering time and $\kappa$ is the associated diffusion coefficient. Not only $\tau_{\text {conf }} \gg \tau_{\text {esc }}$ is required, i.e., $\tau_{\text {sc }} \ll L_{\mathrm{c}} / c$, but also $\tau_{\text {conf }}>L_{\mathrm{c}} / U_{\mathrm{sh}}$, which means that the shock precursor is shorter than the clump $\kappa / U_{\mathrm{sh}} \lesssim L_{\mathrm{CR}}<L_{\mathrm{c}}$ (here $U_{\mathrm{sh}}$ is the shock velocity, and $L_{\mathrm{CR}}$ is the thickness of the CR front near the shock). The last condition ensures that particles with $p_{\|}>p_{1}$ that escape through the clump after having entered it from the shock side, will not interact with the shock after they exit through the opposite side of the clump, thus escaping upstream, Fig 2 The reason for that is a low level of Alfven wave turbulence ahead of the $\mathrm{CR}$ precursor. We also assume that the ambient magnetic field does not deviate strongly from the shock normal, in order to allow these particles to escape through the far side of the clump.

While particles with $p>p_{1}$ escape from the regions of enhanced gamma radiation (high gas density), an initially isotropic distribution of accelerated particles is maintained only in a slab in momentum space $\left|p_{\|}\right|<p_{1}$ and becomes thus highly anisotropic (a 'pancake' distribution). What matters for the integral emission, however, is a locally isotropic component $\bar{f}$ of this new proton distribution. It can be introduced by re-averaging the 'pancake' $\left(\left|p_{\|}\right|<p_{1}\right)$ distribution in pitch angle, $\bar{f}(p) \equiv \int_{0}^{1} f(p, \mu) d \mu$, and is readily obtained assuming that particles remaining in the dense gas (those with $\left|p_{\|}\right|<p_{1}$ ) maintain the flat pitch-angle distribution, i.e.

$$
\bar{f}(p)=\int_{0}^{\mu_{1}} f_{0}(p) d \mu=\left\{\begin{array}{cc}
\left(p_{1} / p\right) f_{0}(p), & p \geq p_{1} \\
f_{0}(p), & p<p_{1}
\end{array}\right.
$$

where $f_{0}(p)$ is the initial (isotropic) distribution function and $\mu_{1}=\min \left\{p_{1} / p, 1\right\}$. Thus, the slope of the particle momentum distribution becomes steeper by exactly one power above $p=p_{1} \equiv p_{\mathrm{br}}$. In particular, any power-law distribution $\propto p^{-q}$, upon entering an $\mathrm{MC}$, turns into $p^{-q-1}$ at $p \geq p_{\mathrm{br}}$, and preserves its form at $p<p_{\mathrm{br}}$.

Note that the broken power-law spectrum can only be maintained if the filling factor $f_{\text {gas }}$ of the dense gas with the significant wave evanescence interval $\left(p_{1}, p_{2}\right)$ is relatively small, $f_{\text {gas }} \ll 1$, so that the overall particle confinement and thus the acceleration are not strongly affected. If, on the contrary, $f_{\text {gas }} \sim 1$, the resonant particles would leak into the $\left(p_{1}, p_{2}\right)$ gap and escape from the accelerator in large amounts, thus suppressing the acceleration. We discuss further limitations of the mechanism in SecVI but we note here that particles with sufficiently high momenta $p>p_{2} B_{0} / \delta B$, where $\delta B / B_{0}$ is the effective mirror ratio of magnetic perturbations, can "jump" over the gap. The primary $p^{-q}$ slope should then be restored for such particles. Recent MAGIC observations of the SNR W51C [26, 31] indeed point at such spectrum recovery at higher energies. It should also be noted, that the $\Delta q=1$ break index is a limiting case of identical gas clumps. The integrated emission from an ensemble of clumps with 


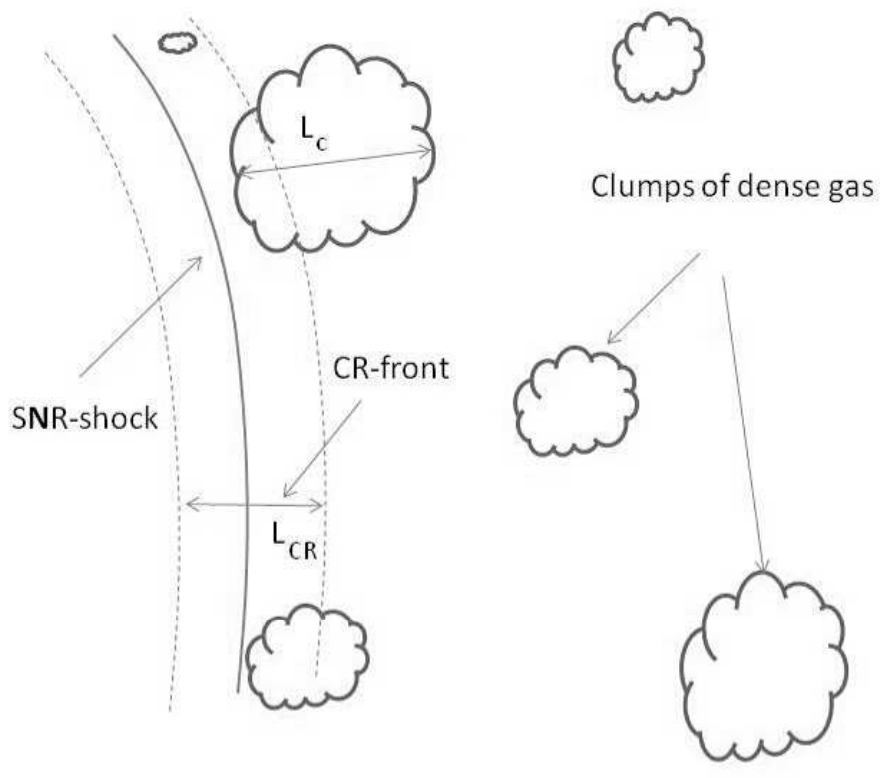

Figure 2: SNR shock propagating into dense gas environment. The filling factor of the gas clumps is small, while some of them may be larger than the thickness of the CR layer near the shock front.

different $p_{1}$ and $p_{2}$ should result in a more complex spectrum.

\section{BREAK MOMENTUM}

While the one power spectral break in the pitch-angle averaged particle distribution seems to be a robust environmental signature of a weakly ionized medium into which the accelerated particles propagate, the break momentum remains uncertain. According to eq.(2), $p_{b r}\left(\equiv p_{1}\right)$ depends on the magnetic field strength and ion density as well as on the frequency of ion-neutral collisions, $v_{i n}=n_{0}\langle\sigma V\rangle$. Here $\langle\sigma V\rangle$ is the product of the collision cross-section and collision velocity averaged over the thermal distribution. Using an approximation of [36, 39] for $\langle\sigma V\rangle, p_{b r}$ can be estimated as

$$
p_{b r} / m c \simeq 10 B_{\mu}^{2} T_{4}^{-0.4} n_{0}^{-1} n_{i}^{-1 / 2}
$$

Here the gas temperature $T_{4}$ is measured in the units of $10^{4} \mathrm{~K}$, magnetic field $B_{\mu}$-in microgauss, $n_{0}$ and $n_{i}$ (number densities corresponding to the neutral/ion mass densities $\rho_{0}$ and $\rho_{i}$ ) -in $\mathrm{cm}^{-3}$. Note that the numerical coefficient in the last expression may vary depending on the average ion and neutral masses and can be higher by a factor of a few [33, 40] than the estimate in eq.(4) suggests. The remaining quantities in the last formula are also known too poorly to make an accurate independent prediction of the position of the break in the gamma ray emission region. Those are the regions near the blast wave where complicated physical processes unfold. They include particle acceleration, strong MHD turbulence (driven by particles and their interaction with ambient gas inhomogeneities), gas ionization by shock generated UV photons, turbulent plasma heating and even evaporation of magnetic cloudlets [39, 41, 42]. Also important may be the ionization by the low energy CRs accelerated at the blast wave. However, as their diffusion length is shorter than that of the particles with $p \gtrsim p_{b r}$, we may assume that they do not reach the MC. Pre-ionization by the UV photons can also be ignored for the column density $N>10^{19} \mathrm{~cm}-2$ ahead of the shock beyond which they are absorbed [29]. The authors or the Ref. [29], using the earlier data from [43] have also analyzed the parameters involved in eq.(4) and found the above estimate of $p_{b r}$ to be in a good agreement with the spectral break position measured by the Fermi LAT. Nevertheless, we may run the argument in reverse and use the Fermi observations [4] of the gamma-ray spectrum of SNR W44 to determine the break momentum in the parent particle spectrum and constrain 


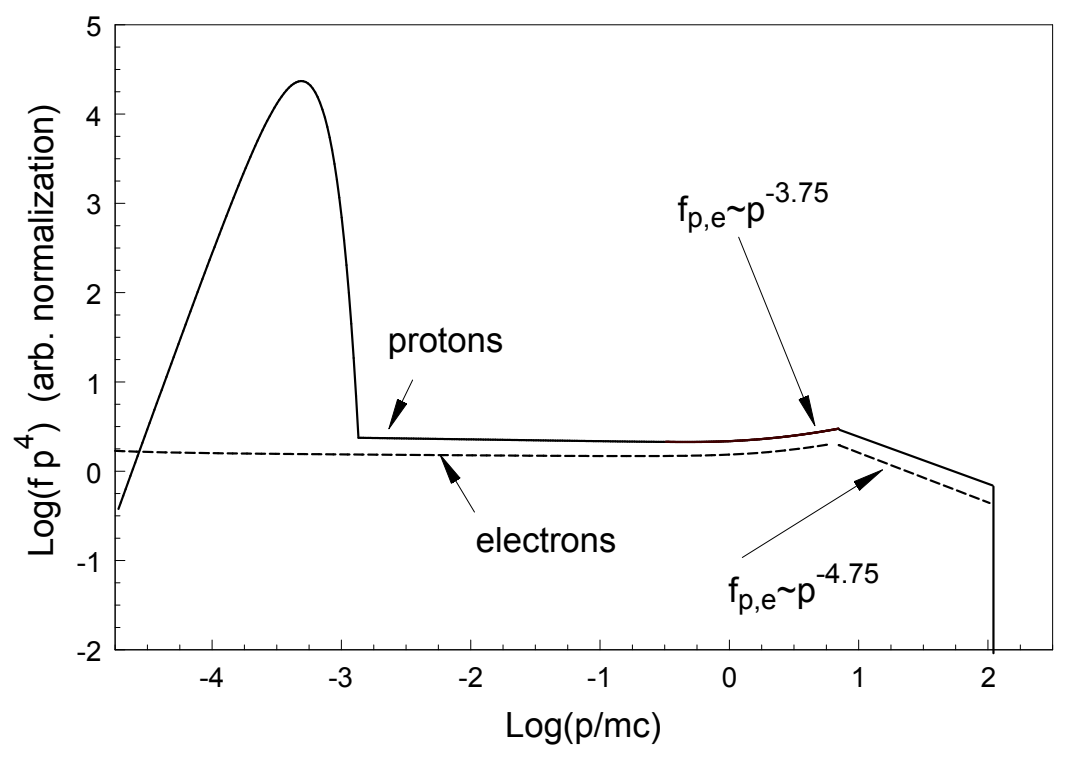

Figure 3: Spectra of accelerated protons and electrons. The both particle distributions are calculated for a weakly modified shock and are shown in momentum normalization $(f(p)$ is steeper by two powers than the spectra in energy normalization, used in the text). Both spectra are multiplied by $p^{4}$, so that the test particle distribution is flat. Shock parameters: acoustic Mach number $M=30$, shock velocity $V_{s} / c=10^{-3}$, the break momentum $p_{b r} \simeq 7 m c$. Shock pre-compression (flow compression across the CR precursor) $\mathrm{R}=1.8$, injection parameter $v \simeq 0$. 1 [defined as $v=(4 \pi / 3)\left(m c^{2} / \rho V_{s}^{2}\right)\left(p_{i n j} / m c\right)^{4} f\left(p_{i n j}\right)$, with $\rho$ and $V_{s}$ being the ambient gas density and the shock speed, respectively]; injection momentum $p_{\text {inj }} / m c \simeq 1.4 \cdot 10^{-3}$.

the parameters in eq.(4). Since we also know the amount of the slope variation $\Delta q$, we can calculate the full spectrum up to the cut-off energy.

It should also be noted that in reality the break at $p=p_{\text {br }}$ is not infinitely sharp for the following reasons. The break momentum may change in space due to variations of the gas parameters (eq. [4]), the resonance broadening [44, 45] near $p=p_{1}=p_{\text {br }}$ (so that particles with $p \gtrsim p_{1}$ are still scattered, albeit weakly) and other factors, such as the contribution of small gas clumps with $L_{c} \ll L_{\mathrm{CR}}$, Fig 2, The small clumps are submerged in the CR front and the CRs that escape from them are readily replenished. Note that this effect may decrease the break index $\Delta q$. However, the conversion of the parent proton spectrum into the observable gamma emission introduces a significant smoothing of the break, so that even a sharply broken proton spectrum produces a smooth gamma spectrum. It provides an excellent fit to the Fermi gamma data without an ad hoc proton break smoothing adopted by the Fermi-team [4] to fit the data. This will be seen from our calculation of the gamma emission based on the sharp proton spectral break in Sec $\nabla$ below. 


\section{PARTICLE SPECTRA}

To calculate the particle spectra, we need to determine the degree of nonlinear modification of the shock structure. In principle, it can be calculated consistently, given the shock parameters and the particle maximum momentum, $p_{\max }$. In the case of a broken spectrum, $p_{b r}$ likely plays the role of $p_{\max }$, as a momentum where the dominant contribution to the pressure of accelerated particles comes from, thus setting the scale of the modified shock precursor. Note that in the conventional nonlinear (NL) acceleration theory, the cut-off momentum $p_{\max }$ plays this role, because the nonlinear spectra are sufficiently flat so as to make the pressure diverge with momentum, unlike broken spectra.

The break in the photon spectrum is observed at about $2 \mathrm{GeV}$, which places the break in the proton distribution at about $p_{b r} \simeq 7 \mathrm{GeV} / c$ [4]. For the strength of the break $\Delta q=1$, the spectrum above it is clearly pressure converging, and we perform the calculation of the shock structure and the spectrum using this break momentum as the point of the maximum in the CR partial pressure. Note that outside of gas clumps the CR pressure may still come from higher momenta and the complete nonlinear calculation of the spectrum would require the filling factor of the gas clumps. However, once the break momentum is set, we can use an analytic approach [13, 46] for a stationary nonlinear acceleration problem using $p_{b r}$ as an input parameter.

Apart from $p_{b r}$, the nonlinear solution depends on a number of other parameters, such as the injection rate of thermal particles into acceleration, Mach number, the precursor heating rate and the shock velocity $V_{s}$. Of these parameters the latter is known reasonably well, $V_{s} \approx 300 \mathrm{~km} / \mathrm{s}$, the injection rate can be either calculated analytically for the parallel shock geometry [47, 48], or inferred from the simulations [49], while the other parameters are still difficult to ascertain. Fortunately, in sufficiently strong shocks the solution either stays close to the test particle (TP) solution (leaving the shock structure only weakly modified) or else it transitions to a strongly modified NL-solution regime. The TP regime typically establishes in cases of moderate Mach numbers, low injection rates and low $p_{\max }$ (now probably closer to $p_{b r}$ ), while the NL regime is unavoidable in the opposite part of the parameter space.

In the TP regime the spectrum is close to a power-law with the spectral index 2 throughout the supra-thermal energy range. In the NL regime, however, the spectrum develops a concave form, starting from a softer spectrum at the injection energy, with the index $q \simeq\left(r_{s}+2\right) /\left(r_{s}-1\right)>2$, where $r_{s}<4$ is the sub-shock compression ratio. Then it hardens, primarily in the region $p \sim m c$, where both the partial pressure and diffusivity of protons change their momentum dependence. The slope reaches its minimum at the cut-off (break) energy, which, depending on the degree of nonlinearity, can be as low as 1.5 or even somewhat lower if the cut-off is abrupt. The question now is into which of these two categories the W44 spectrum falls? Generally, in cases of low maximum (or, equivalently, low spectral break $p_{b r} \lesssim 10$ ) momentum, the shock modification is weak, so the spectrum is more likely to be in a slightly nonlinear, almost TP regime. On the other hand, there is a putative indication from the electron radio emission that their spectrum may be close to $q_{e} \approx 1.75$, which could be the signature of a moderately nonlinear acceleration process. It should be remembered, however, that this is a global index across the W44 remnant. There are resolved bright filaments where a canonical $\alpha=-0.5$ spectrum, corresponding precisely to the TP parent electron spectrum with $q_{e}=2$ is observed [50]. Moreover, there are regions with the positive indices $\alpha \lesssim 0.4$ which cannot be indicative of a DSA process without corrections for subsequent spectral transformations such as an absorption by thermal electrons [50]. These regions can very well contribute to the overall spectral hardening discussed above, mimicking the acceleration nonlinearity. Finally, secondary electrons give rise to the flattening of the radio spectrum as well [29].

If the accelerated protons and electrons respond to the turbulence similarly, which is almost certainly the case in the ultrarelativistic regime, their spectra should have similar slopes there (as long as the synchrotron losses are ignorable). In using the electron radio spectrum as a probe for the level of acceleration nonlinearity, the following two relations are useful. First, there is a relation between the electron energy and the radio frequency $v_{M H z}=4.6 \cdot B_{\mu} E_{G e V}^{2}$. The second, already mentioned relation, $q_{e}=1-2 \alpha$, links the spectral index of radio emission $\alpha$ (assuming the radio flux $\propto v^{\alpha}$ ) and the spectral index of the parent electrons $q_{e}$ (assuming their energy spectrum $\propto E^{-q_{e}}$ ). Once the global radio spectral index of W44, $\alpha \simeq-0.37$ [50] is generated by freshly accelerated electrons in the frequency range $74<v<10700 \mathrm{MHz}$, the electrons should maintain their modified spectrum over the energy range spanning more than one order of magnitude. For example, assuming $B_{\mu} \simeq 70$ [4], one sees that electrons must maintain an index $q_{e} \approx 1.75$ between $0.46<E<5.8 \mathrm{GeV}$. While the upper bound is acceptable given the spectral break proton energy inferred from the super $\mathrm{GeV}$ emission measured by the Fermi LAT, the lower end is rather uncomfortable, since the nonlinear hardening of both protons and electrons with the Bohm (or other similar for protons and electrons turbulent diffusivities) starts (slowly) only at the proton rest energy. The calculated nonlinear spectra are shown in Fig 3 for the both species. At and below $1 \mathrm{GeV}$, the electron spectrum stays close to the test particle solution, $q_{e} \approx 2$, even though the proton spectrum may steepen there, as we mentioned above. The physical reason for this difference is that the electron mean free path falls off slower with decreasing momentum than that of the protons in the Mev-Gev momentum range so that electrons sample longer parts of the shock precursor with higher flow compression and thus develop a harder spectrum. 


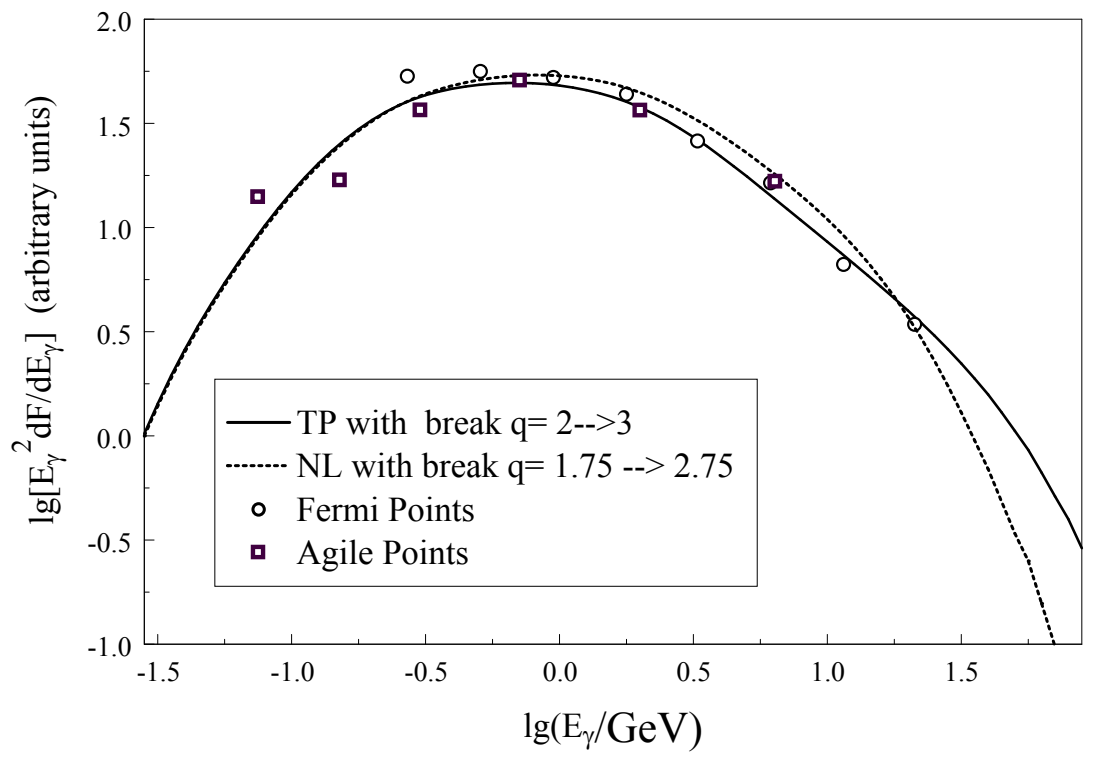

Figure 4: Gamma radiation spectra. Photon spectra resulting from $\pi^{0}$ decay and calculated for two different parent proton spectra compared against the Fermi (circles) and Agile (squares) data. Solid line: a test particle acceleration regime with the spectral index $q=2$ below the break and $q=3$ above the break at $p_{b r}=7 \mathrm{GeV} / \mathrm{c}$. Dashed line: a moderately nonlinear acceleration regime corresponding to the spectrum shown in Fig $3(q \simeq 1.75$ and $q \simeq 2.75$ below and above the break, respectively). Cut-offs are placed at $300 \mathrm{GeV}$ for TP- and $100 \mathrm{GeV}$, for NL-spectrum. Fermi and Agile data are adopted from [4, 9], respectively. Both curves are well within the error bars of Fermi and Agile (not shown for clarity), which, in turn, overlap [9].

\section{PHOTON SPECTRA}

The above considerations somewhat weaken the radio data as a probe for the slope of the electron and (more importantly) for the proton spectrum. Therefore, the exact degree of nonlinearity of the acceleration remains unknown and we consider both the TP and weakly NL regimes in our calculations of the photon spectra, generated in $p-p$ collisions. Specifically, we calculate the $\pi^{0}$ production rate and the gamma-ray emissivity. In so doing, we adopt numerical recipe described in detail in [51, 52]. The physical processes behind these calculations are (i) collisions of accelerated protons with the protons of the ambient gas resulting in the following spectrum of $\pi^{0}$-mesons:

$$
F_{p p}\left(E_{\pi}\right)=4 \pi N_{p g} \int \frac{d \sigma\left(E_{\pi}, E_{p}\right)}{d E_{\pi}} J_{p}\left(E_{p}\right) d E_{p}
$$

where, $N_{p g}$ is the number density of protons in the gas, $d \sigma / d E_{\pi}$ is the differential cross section for the $\pi^{0}$ production in collisions between accelerated protons of energy $E_{p}$ and gas protons, $J_{p}$ is the flux of accelerated protons, $E_{\pi}$ is the energy of $\pi^{0}$ mesons; (ii) decay of $\pi^{0}$ resulting in the gamma emission spectrum 


$$
F\left(E_{\gamma}\right)=2 \int_{E_{\gamma}+m_{\pi}^{2} c^{4} / 4 E_{\gamma}}^{\infty} \frac{F_{p p}\left(E_{\pi}\right)}{\sqrt{E_{\pi}^{2}-m_{\pi}^{2} c^{4}}} d E_{\pi}
$$

where $m_{\pi}$ is the pion rest mass.

The results are shown in Fig 4, The best fit to the Fermi and Agile data is provided by a TP energy distribution $\left(\propto E^{-2}\right)$ below $p_{b r} \simeq 7 \mathrm{GeV} / \mathrm{c}$ with the spectrum steepening by exactly one power above it. The spectrum steepening is perfectly consistent with the proton partial escape described above (with no parameters involved) and shown in Fig 1 For comparison, a weakly NL spectrum, shown in Fig 3, is also used for these calculations (dashed line in Fig, 4), but its fit would require a somewhat stronger break $(\Delta q \gtrsim 1)$ or a low momentum cut-off, Fig 4 i.e. at least one additional free parameter. We will discuss the options in the next section.

\section{SUMMARY AND OUTLOOK}

To summarize the results, the mechanism for a break in the spectrum of shock accelerated protons suggested in [28, 30] is in excellent agreement with the recent [4] Fermi LAT and Agile [9] observations of the SNR W44. The observed gamma ray spectrum most likely results from the decay of $\pi^{0}$-mesons which are born in $p-p$ collisions of shock accelerated protons with an ambient dense gas. The parent proton spectrum is best represented by a classical test particle power law $\propto E^{-2}$, steepening to $E^{-3}$ at $E_{b r} \approx 7 \mathrm{GeV}$ due to deteriorated particle confinement caused by the ion-neutral collisions and the resultant Alfven wave evanescence. The position of the break momentum in the particle spectrum may be estimated using eq. (4), or conversely, the combination of parameters involved in this estimate can be inferred from the measured break momentum. The cut-off momentum is not constrained in this scenario.

An alternative explanation, based on a different mechanism of the break, associated with the change of the particle transport in the CR shock precursor [53] is also possible but is less definitive in the spectrum slope variation $\Delta q$ across the break (see also [29] for the most recent alternative suggestions). In addition, the mechanism [53] would imply a considerable nonlinearity, i.e. a stronger CR shock precompression than that suggested by the radio observation of accelerated electrons and the inferred 100 $\mathrm{GeV}$ proton upper cutoff (see below). Still alternatively, assuming the "environmental" break mechanism is at work, i.e. $\Delta q=1$, but the shock structure is somewhat modified, we arrive at the $E^{-1.75}$ spectrum below the break (as the radio observations may suggest for the electrons), and $E^{-2.75}$ above the break. A fit to the data is marginally possible, but it would require a relatively low cut-off momentum at about $100 \mathrm{GeV} / \mathrm{c}$. This possibility may be supported or ruled out once the data (upper limit) around this energy become available.

As we noted, particle escape from the MC can quench the acceleration process [54]. This would certainly be the case if the MC were filling the entire shock precursor. However, MCs are known to be clumpy [55-58], and fill only a small fraction $(<1-2 \%)$ of the precursor. In this case the acceleration process continues largely unimpeded (apart from the spectrum steepening) but the accelerated protons illuminate the 'cloudlets' and make them visible in $\gamma$-rays due to the high density target material. Another concern is a faint or even lacking $\mathrm{x}$-ray emission that seems to be inconsistent with shocks impacting dense surroundings. This issue has been recently dealt with in, e.g., [58, 59]. Large clumps survive the shock passage as it stalls inside them and no strong heating occurs [58].

The most robust and attractive aspect of the suggested mechanism for the spectral break is the exact $\Delta q=1$ variation of the spectral index. Indeed, this change in the spectral slope is due to the reduction of the number of degrees of freedom of particles caused by the resonant wave evanescence and it does not depend on any parameters. In a combination with the test particle regime operating below the break, which is physically suggested by the low values of the break and upper cut-off momenta, the mechanism provides a very good fit to the Fermi LAT and Agile data with no free parameters for the SNR W44 and probably for W51C. From a number of physically different types of spectral breaks suggested [53, 60-62], namely the current, "environmental" mechanism appears to be plausible where a dense target gas is present which is also required for the efficient $\pi^{0}$ production. However, observations of some other remnants in the dense gas environments, such as W28 and IC443 [6, 7] indicate weaker breaks, $\Delta q=0.6-0.7$ which may either require a different mechanism for the break or a narrower wave evanescence gap $\Delta p=p_{2}-p_{1}$ (higher ionization rate). The predominance of small clumps with $L_{\mathrm{c}} \ll L_{\mathrm{CR}}$ in a MC will also reduce $\Delta q$.

Generally, spectral breaks offer a natural resolution to the well known but puzzling trend of the nonlinear (i.e. supposedly improved) DSA theory to develop spectra which are considerably harder than a simple test particle spectrum, thus becoming even less consistent with the bulk of observations [63, 64]. However, the nonlinear spectrum - i.e., diverging in energy- exhausts the shock energy available for the acceleration as the cut-off momentum grows, so that a broken spectrum should form [53, 61]. Broken spectra are now commonly observed and the old paradigm of a single power-law with an exponential upper cut-off is maladapted to the recent, revolutionarily improved observations [4, 7]. Note, that the spectrum of the RX J1713.7-3946 
[3] is also consistent with the environmental break mechanism presumably operating in W44 surrounding but with a higher $p_{b r} \sim 10^{3} \mathrm{GeV} / \mathrm{c}$ and thus with stronger acceleration nonlinearity [28]. However, it is difficult to make the case for hadronic origin of the gamma-ray emission of the RX J1713.7-3946 [3, 65, 66]. The fundamental role of the W44 remnant for the problem of CR origin is that this particular remnant seems to rule out contaminating electron emission due to Bremsstrahlung and inverse Compton scattering [4, 29] thus favoring the hadronic origin of the gamma emission and bolstering the case for the SNR origin of galactic CRs

\section{Acknowledgments}

We would like to thank the anonymous referee for helpful suggestions. Support by the Department of Energy, Grant No. DE-FG02-04ER54738 is gratefully acknowledged. This work was also supported by the World Class Institute (WCI) Program of the National Research Foundation of Korea (NRF) funded by the Ministry of Education, Science and Technology of Korea (MEST) (NRF Grant Number: WCI 2009-001) 
[1] V. Hess, Physikalische Zeitschrift 13, 1084 (1912).

[2] R. Enomoto, T. Tanimori, T. Naito, T. Yoshida, S. Yanagita, M. Mori, P. G. Edwards, A. Asahara, G. V. Bicknell, S. Gunji, et al., Nature 416, 823 (2002).

[3] F. Aharonian, A. G. Akhperjanian, A. R. Bazer-Bachi, M. Beilicke, W. Benbow, D. Berge, K. Bernlöhr, C. Boisson, O. Bolz, V. Borrel, et al., Astronomy and Astrophys. 449, 223 (2006).

[4] A. A. Abdo, M. Ackermann, M. Ajello, L. Baldini, J. Ballet, G. Barbiellini, M. G. Baring, D. Bastieri, B. M. Baughman, K. Bechtol, et al., Science 327, 1103 (2010).

[5] V. A. Acciari, E. Aliu, T. Arlen, T. Aune, M. Bautista, M. Beilicke, W. Benbow, D. Boltuch, S. M. Bradbury, J. H. Buckley, et al., Astrophys. J. 714, 163 (2010), 1002.2974.

[6] A. A. Abdo, M. Ackermann, M. Ajello, A. Allafort, L. Baldini, J. Ballet, G. Barbiellini, D. Bastieri, K. Bechtol, R. Bellazzini, et al., Astrophys. J. 718, 348 (2010).

[7] A. A. Abdo, M. Ackermann, M. Ajello, L. Baldini, J. Ballet, G. Barbiellini, D. Bastieri, B. M. Baughman, K. Bechtol, R. Bellazzini, et al., Astrophys. J. 712, 459 (2010), 1002.2198.

[8] A. A. Abdo, M. Ackermann, M. Ajello, A. Allafort, L. Baldini, J. Ballet, G. Barbiellini, M. G. Baring, D. Bastieri, R. Bellazzini, et al., Astrophys. J. 734, 28 (2011), 1103.5727.

[9] A. Giuliani, M. Cardillo, M. Tavani, Y. Fukui, S. Yoshiike, K. Torii, G. Dubner, G. Castelletti, G. Barbiellini, A. Bulgarelli, et al., Astrophys. J. Lett. 742, L30 (2011), 1111.4868.

[10] A. A. Abdo, M. Ackermann, M. Ajello, A. Allafort, L. Baldini, J. Ballet, G. Barbiellini, M. G. Baring, D. Bastieri, B. M. Baughman, et al., Astrophys. J. Lett. 710, L92 (2010), 1001.1419.

[11] L. O. Drury, Reports on Progress in Physics 46, 973 (1983).

[12] R. Blandford and D. Eichler, Phys. Rep. 154, 1 (1987).

[13] M. A. Malkov and L. O. Drury, Reports on Progress in Physics 64, 429 (2001).

[14] E. Fermi, Physical Review 75, 1169 (1949).

[15] O. Adriani, G. C. Barbarino, G. A. Bazilevskaya, R. Bellotti, M. Boezio, E. A. Bogomolov, L. Bonechi, M. Bongi, V. Bonvicini, S. Borisov, et al., Science 332, 69 (2011), 1103.4055.

[16] H. S. Ahn, P. Allison, M. G. Bagliesi, J. J. Beatty, G. Bigongiari, J. T. Childers, N. B. Conklin, S. Coutu, M. A. DuVernois, O. Ganel, et al., Astrophys. J. Lett. 714, L89 (2010), 1004.1123.

[17] A. D. Panov, J. H. Adams, H. S. Ahn, G. L. Bashinzhagyan, J. W. Watts, J. P. Wefel, J. Wu, O. Ganel, T. G. Guzik, V. I. Zatsepin, et al., Bulletin of the Russian Academy of Science, Phys. 73, 564 (2009), 1101.3246.

[18] J. Alcaraz, B. Alpat, G. Ambrosi, H. Anderhub, L. Ao, A. Arefiev, P. Azzarello, E. Babucci, L. Baldini, M. Basile, et al., Physics Letters B 490, 27 (2000).

[19] K. Koyama, R. Petre, E. V. Gotthelf, U. Hwang, M. Matsuura, M. Ozaki, and S. S. Holt, Nature 378, 255 (1995).

[20] G. E. Allen, R. Petre, and E. V. Gotthelf, Astrophys. J. 558, 739 (2001).

[21] M. A. Malkov, P. H. Diamond, and R. Z. Sagdeev, Physical Review Letters 108, 081104 (2012), 1110.5335.

[22] A. E. Vladimirov, G. Jóhannesson, I. V. Moskalenko, and T. A. Porter, ArXiv e-prints 1108.1023 (2011), 1108.1023.

[23] Y. Ohira and K. Ioka, Astrophys. J. Lett. 729, L13+ (2011), 1011.4405.

[24] P. Blasi and E. Amato, ArXiv e-prints 1105.4521 (2011), 1105.4521.

[25] T. Tanaka, Y. Uchiyama, F. A. Aharonian, T. Takahashi, A. Bamba, J. S. Hiraga, J. Kataoka, T. Kishishita, M. Kokubun, K. Mori, et al., Astrophys. J. 685, 988 (2008), 0806.1490.

[26] E. Carmona, J. Krause, I. Reichardt, and for the Magic Collaboration, Probing proton acceleration in W51C with MAGIC (2011), 1110.0950.

[27] O. Reimer and M. Pohl, Astronomy and Astrophys. 390, L43 (2002).

[28] M. A. Malkov, P. H. Diamond, and R. Z. Sagdeev, Astrophys. J. Lett. 624, L37 (2005).

[29] Y. Uchiyama, R. D. Blandford, S. Funk, H. Tajima, and T. Tanaka, Astrophys. J. Lett. 723, L122 (2010).

[30] M. A. Malkov, P. H. Diamond, and R. Z. Sagdeev, Nature Communications 2/1195 (2011), 1004.4714.

[31] J. Aleksić, E. A. Alvarez, L. A. Antonelli, P. Antoranz, M. Asensio, M. Backes, U. Barres de Almeida, J. A. Barrio, D. Bastieri, J. Becerra González, et al., Astronomy and Astrophys. 541, A13 (2012), 1201.4074.

[32] A. Neronov, D. V. Semikoz, and A. M. Taylor, Physical Review Letters 108, 051105 (2012), 1112.5541.

[33] R. Kulsrud and W. P. Pearce, Astrophys. J. 156, 445 (1969).

[34] E. G. Zweibel and J. M. Shull, Astrophys. J. 259, 859 (1982).

[35] H. J. Völk, G. E. Morfill, and M. A. Forman, Astrophys. J. 249, 161 (1981).

[36] L. O. C. Drury, P. Duffy, and J. G. Kirk, Astronomy and Astrophys. 309, 1002 (1996).

[37] B. Reville, J. G. Kirk, P. Duffy, and S. O'Sullivan, International Journal of Modern Physics D 17, 1795 (2008).

[38] J. Skilling, Mon. Not. R. Astron. Soc. 172, 557 (1975).

[39] B. T. Draine and C. F. McKee, ARA\&A 31, 373 (1993).

[40] T. Nakano, Fundamentals of Cosmic Physics 9, 139 (1984).

[41] J. M. Shull and C. F. McKee, Astrophys. J. 227, 131 (1979).

[42] A. M. Bykov, R. A. Chevalier, D. C. Ellison, and Y. A. Uvarov, Astrophys. J. 538, 203 (2000).

[43] W. T. Reach, J. Rho, and T. H. Jarrett, Astrophys. J. 618, 297 (2005).

[44] T. H. Dupree, Physics of Fluids 9, 1773 (1966), URL http://link .aip.org/link/?PFL/9/1773/1

[45] A. Achterberg, Astronomy and Astrophys. 98, 161 (1981). 
[46] M. A. Malkov, Astrophys. J. 485, 638 (1997).

[47] M. A. Malkov and H. J. Völk, Astronomy and Astrophys. 300, 605 (1995).

[48] M. A. Malkov, Phys. Rev. E 58, 4911 (1998).

[49] L. Gargaté and A. Spitkovsky, Astrophys. J. 744, 67 (2012), 1107.0762.

[50] G. Castelletti, G. Dubner, C. Brogan, and N. E. Kassim, Astronomy and Astrophys. 471, 537 (2007).

[51] T. Kamae, N. Karlsson, T. Mizuno, T. Abe, and T. Koi, Astrophys. J. 647, 692 (2006).

[52] N. Karlsson and T. Kamae, Astrophys. J. 674, 278 (2008).

[53] M. A. Malkov and P. H. Diamond, Astrophys. J. 642, 244 (2006).

[54] L. O. Drury, Mon. Not. R. Astron. Soc. 415, 1807 (2011), 1009.4799.

[55] R. M. Crutcher, Astrophys. J. 520, 706 (1999).

[56] R. A. Chevalier and J. Oishi, Astrophys. J. Lett. 593, L23 (2003).

[57] E. Parizot, A. Marcowith, E. van der Swaluw, A. M. Bykov, and V. Tatischeff, Astronomy and Astrophys. 424, 747 (2004).

[58] T. Inoue, R. Yamazaki, S.-i. Inutsuka, and Y. Fukui, Astrophys. J. 744, 71 (2012), 1106.3080.

[59] L. Drury, F. A. Aharonian, D. Malyshev, and S. Gabici, Astronomy and Astrophys. 496, 1 (2009), 0811.3566.

[60] F. A. Aharonian and A. M. Atoyan, Astronomy and Astrophys. 309, 917 (1996).

[61] M. A. Malkov, P. H. Diamond, and T. W. Jones, Astrophys. J. 571, 856 (2002).

[62] Y. Ohira, K. Murase, and R. Yamazaki, Mon. Not. R. Astron. Soc. 410, 1577 (2011), 1007.4869.

[63] T. K. Gaisser, R. J. Protheroe, and T. Stanev, Astrophys. J. 492, 219 (1998).

[64] A. M. Hillas, Journal of Physics G Nuclear Physics 31, 95 (2005).

[65] F. A. Aharonian, A. G. Akhperjanian, K.-M. Aye, A. R. Bazer-Bachi, M. Beilicke, W. Benbow, D. Berge, P. Berghaus, K. Bernlöhr, O. Bolz, et al., Nature 432, 75 (2004).

[66] B. Katz and E. Waxman, Journal of Cosmology and Astro-Particle Physics 1, 1 (2008). 